\title{
Dissociation between Neuronal Activity in Sensorimotor Cortex and Hand Movement Revealed as a Function of Movement Rate
}

\author{
Dora Hermes, ${ }^{1}$ Jeroen C. W. Siero, ${ }^{1}$ Erik J. Aarnoutse, ${ }^{1}$ Frans S. S. Leijten, ${ }^{1}$ Natalia Petridou, ${ }^{1,2}$ and Nick F. Ramsey ${ }^{1}$ \\ ${ }^{1}$ Rudolf Magnus Institute of Neuroscience, Department of Neurology and Neurosurgery, Section of Brain Function and Plasticity and ${ }^{2}$ Department of \\ Radiology, University Medical Center Utrecht, 3584 CX Utrecht, The Netherlands
}

It is often assumed that similar behavior is generated by the same brain activity. However, this does not take into account the brain state or recent behavioral history and movement initiation or continuation may not be similarly generated in the brain. To study whether similar movements are generated by the same brain activity, we measured neuronal population activity during repeated movements. Three human subjects performed a motor repetition task in which they moved their hand at four different rates $(0.3,0.5,1$, and $2 \mathrm{~Hz})$. From high-resolution electrocorticography arrays implanted on motor and sensory cortex, high-frequency power $(65-95 \mathrm{~Hz})$ was extracted as a measure of neuronal population activity. During the two faster movement rates, high-frequency power was significantly suppressed, whereas movement parameters remained highly similar. This suppression was nonlinear: after the initial movement, neuronal population activity was reduced most strongly, and the data fit a model in which a fast decline rapidly converged to saturation. The amplitude of the beta-band suppression did not change with different rates. However, at the faster rates, beta power did not return to baseline between movements but remained suppressed. We take these findings to indicate that the extended beta suppression at the faster rates, which may suggest a release of inhibition in motor cortex, facilitates movement initiation. These results show that the relationship between behavior and neuronal activity is not consistent: recent movement influences the state of motor cortex and facilitates next movements by reducing the required level of neuronal activity.

\section{Introduction}

In daily life, we often make series of similar movements, such as knocking on a door, walking, and clapping our hands, but the exact same movements can also be made once. It is often assumed that one action results from a specific pattern of neuronal activity. In motor cortex, this would assume that similar, repeated movements are induced by the same amount and pattern of motor cortex activity. However, behavioral and functional imaging studies have discussed whether a single movement is generated in the same way when it is performed in a series of repetitions (Miall and Ivry, 2004; Schaal et al., 2004; Levy-Tzedek et al., 2011). Specific problems with movement initiation or perseveration in neurological disorders such as Parkinson's disease suggest that,

Received Jan. 15, 2012; revised April 29, 2012; accepted May 31, 2012.

Author contributions: D.H., J.C.W.S., N.P., and N.F.R. designed research; D.H., J.C.W.S., E.J.A., F.S.S.L., and N.P. performed research; D.H. and E.J.A. contributed unpublished reagents/analytic tools; D.H. analyzed data; D.H., J.C.W.S., N.P., and N.F.R. wrote the paper.

This research is supported by the Dutch Technology Foundation, the Applied Science Division of the Dutch Foundation for Scientific Research and the Technology Program of the Ministry of Economic Affairs, and University of Utrecht Grant UGT7685. We thank Eb Fetz, Kai Miller, and Peter Brunner for their valuable comments and proofreading of this manuscript, Mariska Vansteensel, Martin Bleichner, Cyrille Ferrier, Geertjan Huiskamp, Herke Jan Noordmans, and Tineke Gebbink for their help in collecting the data, Peter Gosselaar and Peter van Rijen for implanting the electrodes and the staff of the clinical neurophysiology department for their time and effort.

Correspondence should be addressed to Nick F. Ramsey and Dora Hermes, Department of Neurology and Neurosurgery, Heidelberglaan 100, G03.124, 3584 CX Utrecht, The Netherlands. E-mail: n.f.ramsey@umcutrecht.nl or d.hermes@umcutrecht.nl.

DOI:10.1523/JNEUROSCI.0357-12.2012

Copyright $\odot 2012$ the authors $\quad 0270-6474 / 12 / 329736-09 \$ 15.00 / 0$ during repeated movements, not all movements are similarly generated (Ebersbach et al., 1994; Ridley, 1994; Praamstra et al., 1998; Redgrave et al., 2010). The assumption of a one-to-one correspondence between action and motor cortex activity can therefore be questioned.

The beta rhythm decreases during motor tasks (Jasper and Andrews, 1938; Pfurtscheller, 1981), and EEG research has shown that beta rhythm activity does not return to baseline between fast movements but remains suppressed (Toma et al., 2002). Increased amplitude of the sensorimotor beta rhythm is related to decreased corticospinal excitability and difficulty to induce hand movements by motor cortex stimulation (Hummel et al., 2002). Also, increased beta power is related to successfully stopping unwanted actions (Swann et al., 2009). This suggests that, during increased beta power, motor cortex is inhibited. These studies may thus indicate that, when beta power is suppressed during fast, repetitive action, the motor cortex is continuously released from inhibition. Movements have also been linked to transient increases in high-frequency power of $>60 \mathrm{~Hz}$ (Crone et al., 1998; Miller et al., 2007) associated with local processing in motor cortex. Whether the release from inhibition during repeated movement is also linked to a different level of the actual local neuronal activity that is associated with a movement is yet unknown.

We recorded brain signals from high-resolution implanted electrode grids in epilepsy patients [electrocorticography (ECoG)] from motor and somatosensory cortex and 
Table 1. Behavioral results

\begin{tabular}{llllll}
\hline \multirow{2}{*}{$\begin{array}{lllll}\text { Reaction times } \\
\text { Subject }\end{array}$} & \multicolumn{4}{l}{ Number of movements } \\
\cline { 3 - 6 } & {$[$ mean \pm SD (ms) $]$} & $\begin{array}{l}0.3 \mathrm{~Hz} \\
\text { One movement }\end{array}$ & $\begin{array}{l}0.5 \mathrm{~Hz} \\
\text { (3 cues) }\end{array}$ & $\begin{array}{l}1 \mathrm{~Hz} \\
\text { (7 cues) }\end{array}$ & $\begin{array}{l}2 \mathrm{~Hz} \\
\text { (13 cues) }\end{array}$ \\
\hline 1 & $404 \pm 77$ & 3 & 4 & 7.9 & 13.5 \\
2 & $577 \pm 73$ & 2.9 & 3.9 & 4.8 & 11.2 \\
3 & $445 \pm 77$ & 3 & 4 & 7 & 9.2 \\
\hline
\end{tabular}

Reaction times during the first task in which patients made one hand movement. For the repetition tasks, the average number of hand movements for each trial is shown. Note that patients started to miss cues or make too many movements (holding their own pace) with movement rates of 1 and $2 \mathrm{~Hz}$.

pressed between movements, and high-frequency power in motor cortex was suppressed after the initial movement. These data suggest that the correspondence between neuronal activity in sensorimotor cortex and hand movement changes with movement rate.

\section{Materials and Methods}

Subjects and procedure. Three right-handed patients (one female, two males; mean age, 27 years; range, $19-43$ years) who were scheduled for the implantation of ECoG arrays for the clinical purpose of epilepsy monitoring gave informed consent to participate in this study. These patients had normal

A
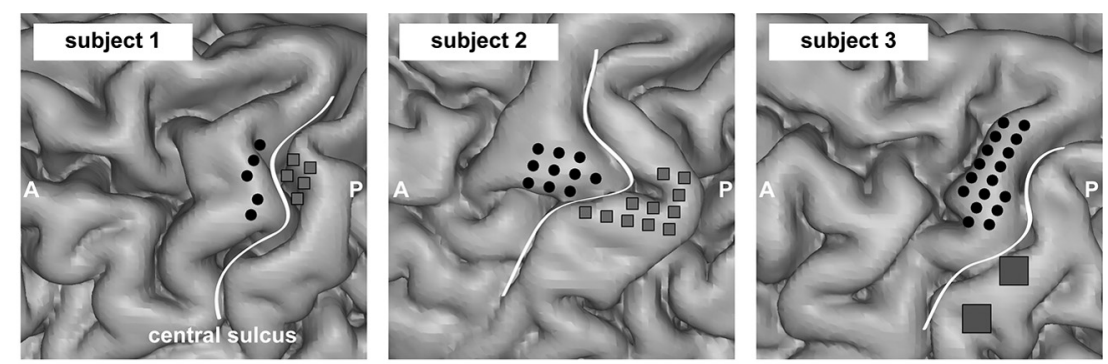

$\mathrm{B}$
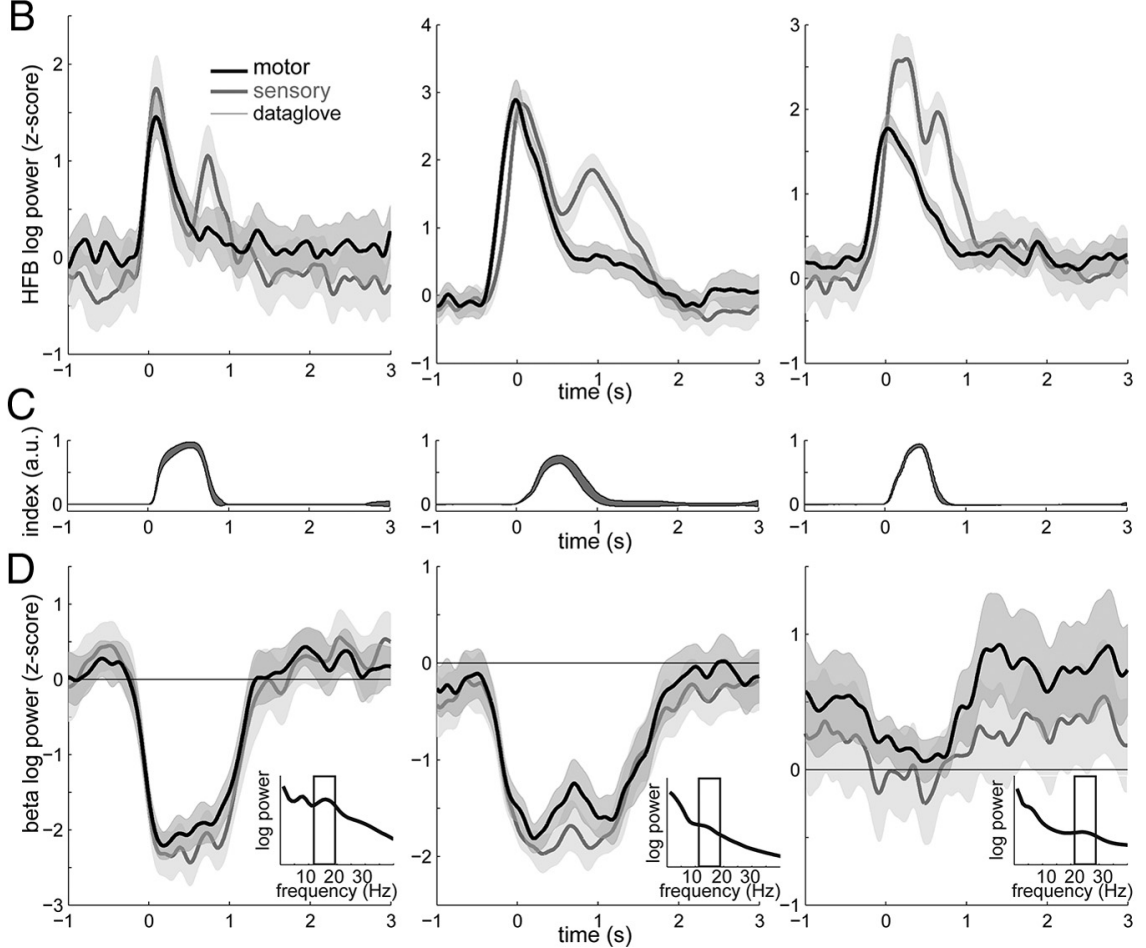

Figure 1. Responses in HFB and beta power on motor cortex and sensory cortex. $\boldsymbol{A}$, The distribution of electrodes on motor and sensory cortex ( $A$, anterior; $P$, posterior). Subject 3 did not have coverage of high-resolution electrodes on sensory cortex, and larger electrodes from the regular grid were selected. $\boldsymbol{B}$, The HFB response during movement onset at $0 \mathrm{~ms}$ averaged for electrodes on motor cortex (black) and sensory cortex (gray); light gray areas indicate \pm 3 SE. C, The index finger movement from the data glove. $D$, Beta response after movement onset. The small inset shows the power spectra during the task, which were used to define the indicated beta peak ( $12-18 \mathrm{~Hz}$ for subjects 1 and 2 and $22-28 \mathrm{~Hz}$ for subject 3 ). a.u., Arbitrary units.

calculated beta power $(12-28 \mathrm{~Hz})$ and high-frequency band activity (HFB; 65-95 Hz). Broadband high-frequency activity is associated with neuronal population firing rate (Manning et al., 2009; Miller et al., 2009a; Ray and Maunsell, 2011) and correlates well with the fMRI signal (Hermes et al., 2011). We hypothesize that, during fast, repeated movements, when the motor cortex is presumably in a continuously disinhibited state, the amount of high-frequency activity related to repeated movements will change. Three subjects made movements at four rates $(0.3 .0 .5,1$, and $2 \mathrm{~Hz}$ ). During the fastest rates, beta power remained sup- hand function, and the pathological region did not extend to sensorimotor cortex. The study was approved by the ethical committee of the University Medical Center Utrecht, in accordance with the Declaration of Helsinki 2008. On average, 125 electrodes were implanted, of which 32 in each subject were high-resolution mini-grids of $8 \times 4$ electrodes located on left sensorimotor cortex. Mini-grids had an interelectrode spacing of $3.3 \mathrm{~mm}$ center to center and a spherical measurement surface of $1 \mathrm{~mm}$ diameter. To localize the electrodes with high accuracy, we used the method presented previously (Hermes et al., 2010), in which electrodes were localized from a high-resolution computed tomography scan (Philips Tomoscan SR7000) and projected to the cortical surface rendering generated from a preoperative MRI scan of each subject (Philips 3T Achieva).

Experimental paradigm. The experiment consisted of two parts, the first to map ECoG responses during a single movement (see Fig. 1 ) and the second to test repeated movement effects (see Fig. 2). The single-movement part started and ended with a $30 \mathrm{~s}$ rest period during which a red circle was presented on a computer screen and subjects relaxed with their right hand open, palm facing up. After the first $30 \mathrm{~s}$ rest, the circle turned green 54 times for $500 \mathrm{~ms}$ at intertrial intervals varying from 3 to $18 \mathrm{~s}$, with an average of $8 \mathrm{~s}$. When the circle turned green, the subjects closed and opened their right hand.

The repeated-movement part consisted of four movement repetition tasks in which subjects moved at four different rates $(0.33,0.5,1$, and $2 \mathrm{~Hz}$; see Fig. $2 \mathrm{~A}$ ). In the first task, each trial consisted of three visual cues (the red circle turning green similar as in the first part, for $400 \mathrm{~ms}$ ), presented at $3 \mathrm{~s}$ intercue interval, instructing the subject to close and open their hand, thus resulting in $0.33 \mathrm{~Hz}$ movement. In the second, third, and fourth tasks, respectively, 4, 7, or 13 cues were given with 2,1 , and 0.5 s intercue interval. Each trial was followed by a rest period until a total trial duration of $19 \mathrm{~s}$ was reached.

Each task consisted of 13-15 trials of $19 \mathrm{~s}$.

Behavioral measurement and analysis. Finger movements were measured using a five degrees of freedom data glove (5DT Inc.). The dataglove data were recorded simultaneously with the ECoG data using a custom workspace extension in Presentation (Neurobehavioral Systems). The onset of each movement was visually detected; there were drifts in the data-glove data, and assigning a simple threshold to detect movement onsets was not sufficiently accurate. Therefore, movement onsets were visually detected as the first deviation from the premovement baseline for optimal accuracy. To extract a measure for whole-hand 


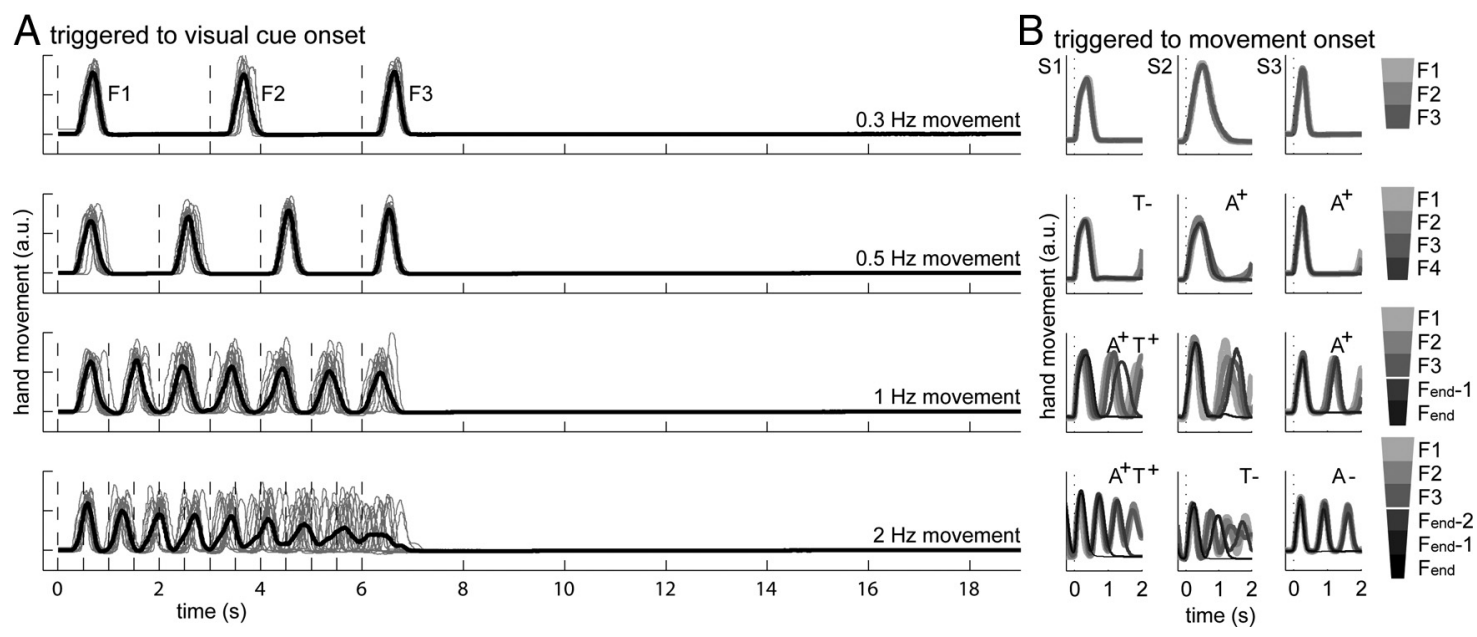

Figure 2. Repetition task design and behavioral responses. $A$, Hand movement during the four different repetition tasks as measured with a data glove. The top shows that, in the first task, three visual cues (dashed lines) were given to open and close the hand with 3 sintercue intervals $(\sim 0.3 \mathrm{~Hz})$. The other panels show tasks $2-4$, respectively, and subjects opened and closed their hand at $\sim 0.5, \sim 1$, and $\sim 2 \mathrm{~Hz}$. In each trial, a series of $3,4,7$, or 13 cues was followed by a rest period until $19 \mathrm{~s}$. The average response is shown in black; individual trials are shown in gray for trials in which $>2,>3,>5$, and $>7$ responses were made. Note that, for the 1 and $2 \mathrm{~Hz}$ movements, this patient started to make mistakes: movements were made too early or too late and cues were missed. Therefore, in the average response (black), it seems as if movements were smaller, whereas the individual trials do not show this effect. $\boldsymbol{B}$, To resolve this apparent reduction in movement response size, trials were triggered with respect to movement onsets $(t=$ 0 , dotted lines). The grayscale indicates the hand flexion number (F). For the three different subjects (S1-S3), the average index finger movement for the first, second, and third and the first, second, third, and fourth responses are shown for the 0.3 and $0.5 \mathrm{~Hz}$ tasks. Because mistakes started to be made for the 1 and $2 \mathrm{~Hz}$ tasks, these show the first, second, third, next to last (end-1), and last (end) response and the first, second, third, second to last (end-2), next to last (end-1), and last (end) response. All plots for the same subject have the same scale. Note that, when index finger movement is locked to response onset, the amplitude for subsequent movements in one task remains similar. At the faster rates with intercue interval of $\angle 2 \mathrm{~s}$, the HFB responses to the subsequent $s$ timuli are also visible, but because these are locked to the previous hand movement and not to their response, they are not relevant for analyses. An A or T indicates significant effects of flexion number on movement amplitude or speed (time to maximum flexion); + or - symbol indicates an increase or decrease in either A or T with flexion number. a.u., Arbitrary units.

movement in addition to each individual finger, we used the first component from a principal component analysis on all five channels. The movement amplitude and speed of flexion were calculated as follows. The average baseline was subtracted from each trial to correct for baseline offset. Hand-movement amplitude was defined as the maximum deviation (when the hand is completely closed) within $500 \mathrm{~ms}$ after movement onset. The speed of flexion for each movement was defined as the lag between movement onset and the maximum deviation.

ECoG acquisition and analysis. ECoG data were acquired with a 128 channel recording system (Micromed) with $512 \mathrm{~Hz}$ sampling rate and $0.15-134.4 \mathrm{~Hz}$ bandpass filter. Data were re-referenced to the common average of all electrodes recorded from the same amplifier (including electrodes on the regular grids to get a better estimate for the background noise for subjects 1 and 2). To extract power changes in high frequencies and the beta band, ECoG data were filtered for high frequencies (65-95 $\mathrm{Hz}$ ) and for the beta band using a third-order Butterworth filter in two directions to minimize phase distortion (using the filtfilt function in MATLAB; MathWorks). The beta band was defined based on peaks in the power spectrum from the first single-movement task (see Fig. 1) and ran from 12 to $18 \mathrm{~Hz}$ for subjects 1 and 2 and from 22 to $28 \mathrm{~Hz}$ for subject 3. It can be noted that this peak differs between subjects; this may be attributable to simple individual differences or long-time use of antiepileptic drugs (van Beijsterveldt et al., 1996; Jensen et al., 2005). After filtering, the log power of the analytic amplitude (by Hilbert transform) was calculated and the signal was smoothed with a $250 \mathrm{~ms}$ Gaussian window ( $42 \mathrm{~ms} \mathrm{SD}$ ). The smoothed log power from the first task was then normalized ( $z$-score) with respect to the mean and SD of $20 \mathrm{~s}$ rest before the start of the task. The log power from the repetition tasks was normalized ( $z$-score) with respect to the mean and SD of all $15-19$ s periods at the end of the trials. This baseline was thus the same for all trials within a task, and potential changes with repetition cannot be attributed to differences in the baseline.

To map basic ECoG responses on sensory and motor areas, we selected electrodes on motor and sensory areas based on anatomical boundaries (the central sulcus and precentral and postcentral sulci); electrodes located over a sulcus were not included (see Fig. $1 A$ ). The $z$-scored log power was then averaged across electrodes on motor cortex and sensory cortex.
Subsequently, we studied the effects of repetition and movement rate on behavioral data and beta and HFB amplitude. Using a repeatedmeasures ANOVA, we tested for significant changes in movement amplitude and speed (data-glove onset to maximum flexion), beta amplitude, and HFB amplitude across repeated movements within a task. The repeat factor was movement number: for task $1(0.3 \mathrm{~Hz})$, flexion numbers 1,2 , and 3 ; for tasks $2-4(0.5,1$, and $2 \mathrm{~Hz})$, flexion numbers 1 , 2,3 , and the last flexion. Because adding more data points for the fast movements might bias the chance of the ANOVA showing significant changes across responses, we kept the number of data points in the ANOVA the same across the different movement speeds and only used the first three plus the last response for all tasks. Because there were three subjects, statistics from individual subjects are reported. Results are considered significant if statistics showed significant effects in all three subjects (at $p<0.05$ ) and effects were in the same direction.

\section{Results}

\section{ECoG responses in sensory and motor cortex during a} single movement

In the first part of the experiment, subjects performed a task in which a single hand movement was made to localize and map basic ECoG responses. The subjects' average \pm SD reaction time was $475 \pm 90 \mathrm{~ms}$ across the three subjects (Table 1 ). The ECoG signal was filtered for HFB and the beta band. Figure 1 shows the HFB and beta response for electrodes on motor cortex and sensory cortex and the average behavioral response from the index finger of the data glove. On average, HFB responses in motor cortex reached $50 \%$ of the peak amplitude $147 \pm 91 \mathrm{~ms}$ before movement onset and in sensory cortex $60 \pm 51 \mathrm{~ms}$ before movement onset. Previous ECoG studies have observed similar lags between high-frequency increase and movement onset (Kubánek et al., 2009; Miller et al., 2009b). Surprisingly, the first sensory response starts before the onset of movement. Also note that, although in motor cortex there is only one peak in the HFB response, in sensory cortex there are two subsequent peaks. 
A
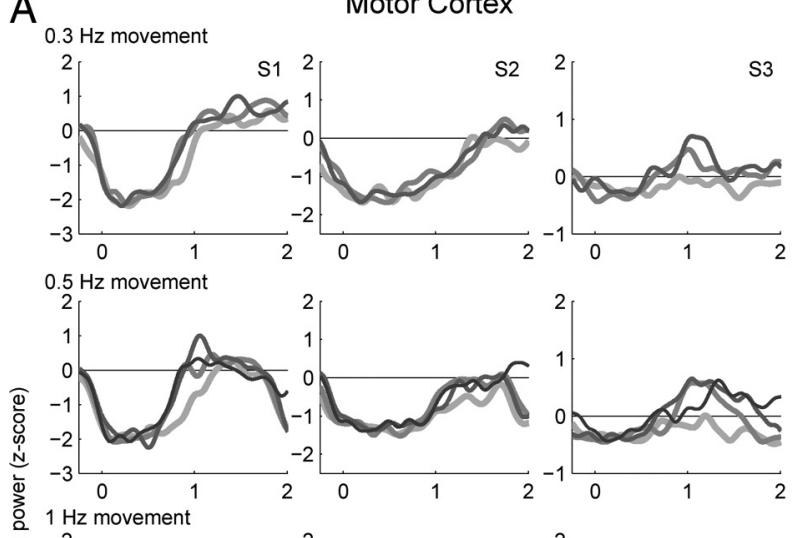

음

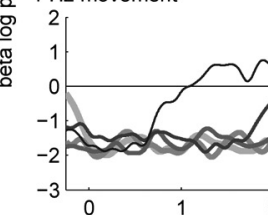

$2 \mathrm{~Hz}$ movement

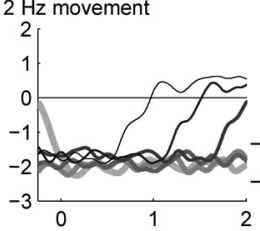

Motor Cortex
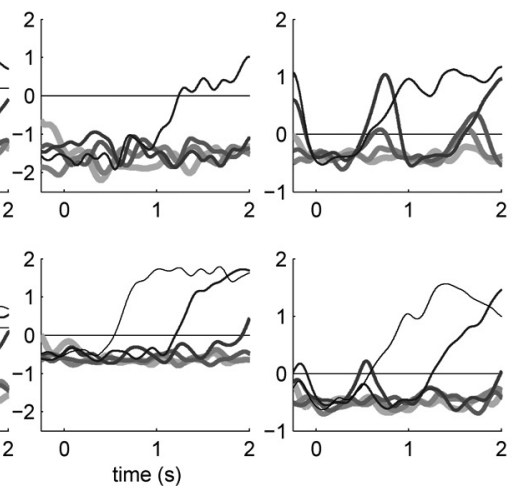

B
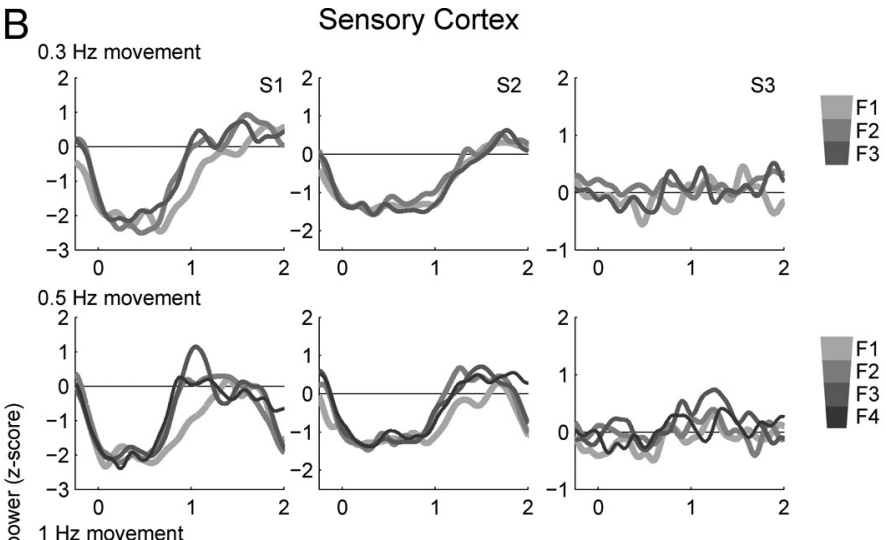
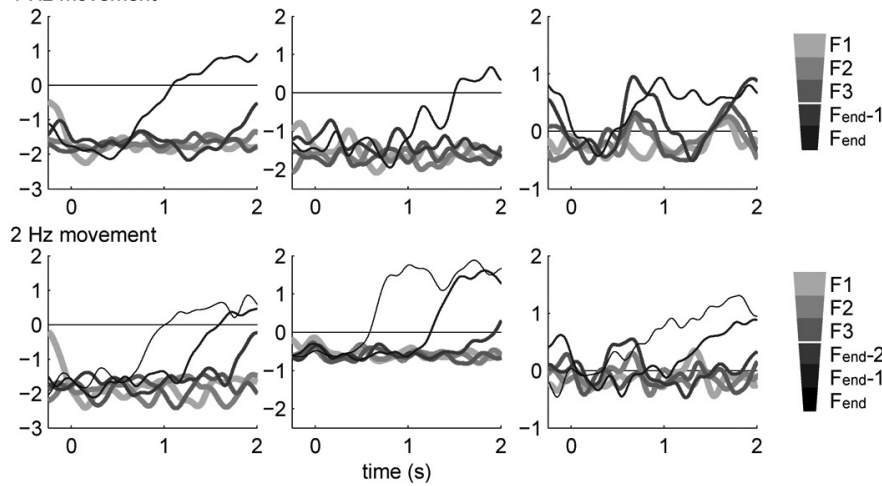

Figure 3. Beta responses on motor cortex and sensory cortex. A, Beta responses on motor cortex as a function of time after movement onset for subjects 1-3 (S1-S3) from left to right and during the different movement rates from top to bottom. Note that, at the faster rates with intercue interval of $<2 \mathrm{~s}$, the beta responses to the subsequent stimuli are also visible. The grayscale indicates the hand flexion number (F) as in Figure 2. B, Beta responses on sensory cortex for subjects 1-3 from left to right and during the different movement rates from top to bottom.

\section{Behavioral data during movement repetition}

Hand movement was measured with a data glove during the movement repetition task. Table 1 shows that, during 0.33 and $0.5 \mathrm{~Hz}$ movement, the subjects followed the cues, and when shown three or four cues, they made three and four movements on average, respectively. During the faster movements of 1 and $2 \mathrm{~Hz}$, behavior changed: they sometimes moved before a cue or missed a cue. This indicates that they were moving more continuously at their own pace. As a result, when seven cues were given at $1 \mathrm{~Hz}$, the three subjects made eight, five, or seven movements on average; when 13 cues were given at $2 \mathrm{~Hz}$, they made 14, 11, and 9 movements. Using cues as onsets will thus blur the data, and we thus used movement onset, which is far more precise. Figure $2 \mathrm{~A}$ demonstrates this effect in the data-glove responses for subject 3 during the four different tasks.

Next, we tested whether subsequent movements within a task differed in movement amplitude or speed (onset to maximum flexion). Figure $2 B$ shows the average hand movement for the three subjects for flexion numbers 1,2, and 3 from task $1(0.3 \mathrm{~Hz})$, flexion numbers $1,2,3$, and 4 from task $2(0.5 \mathrm{~Hz})$, flexion numbers 1, 2, 3, and the last two flexions from task 3 ( 1 $\mathrm{Hz}$ ), and flexion numbers $1,2,3$, and the last three flexions from task 4 (2 Hz). Repeated movements look highly similar. To test for effects of repetition on movement size and speed within a task, a repeated-measures ANOVA was performed. No significant changes in movement amplitude or speed were noted that were consistent across the three subjects (Fig. $2 \mathrm{~B}$ shows the results of the ANOVA for each individual subject).

\section{Changes in the beta and HFB response with repeated movement}

First, we studied the effect of movement rate on beta power. Figure 3 shows the average beta response during the movement repetition task in motor cortex and sensory cortex for all three subjects. It can be seen that, at the faster movement rates ( 1 and 2 $\mathrm{Hz}$ ), the beta band activity remains continuously suppressed: power does not return to baseline after every individual movement but only after the last movement of a series.

To test whether there were any significant changes in beta amplitude with repeated movement within one task, the beta power was averaged from 0 to $300 \mathrm{~ms}$ after movement onset (time window chosen based on Fig. 1D). A repeated-measures ANOVA on beta amplitude for each task showed no significant effect of repetition on beta amplitude across the three subjects, indicating that the amplitude change was the same regardless of movement rate.

Second, we then studied the effect of movement rate on highfrequency power. High-frequency power was extracted from electrodes on motor and sensory cortex as a measure of local neuronal activity. Figure $4, A$ and $B$, shows the average HFB response in motor and sensory cortex during the different movement repetition tasks. It can be seen that, during the faster movements of 1 and $2 \mathrm{~Hz}$, the first HFB response is larger than the subsequent responses. Note that this effect is most pronounced in motor cortex.

To test whether this change in HFB activity with repeated movements was significant, the HFB power was averaged from -100 to $300 \mathrm{~ms}$ around movement onset (time window chosen 


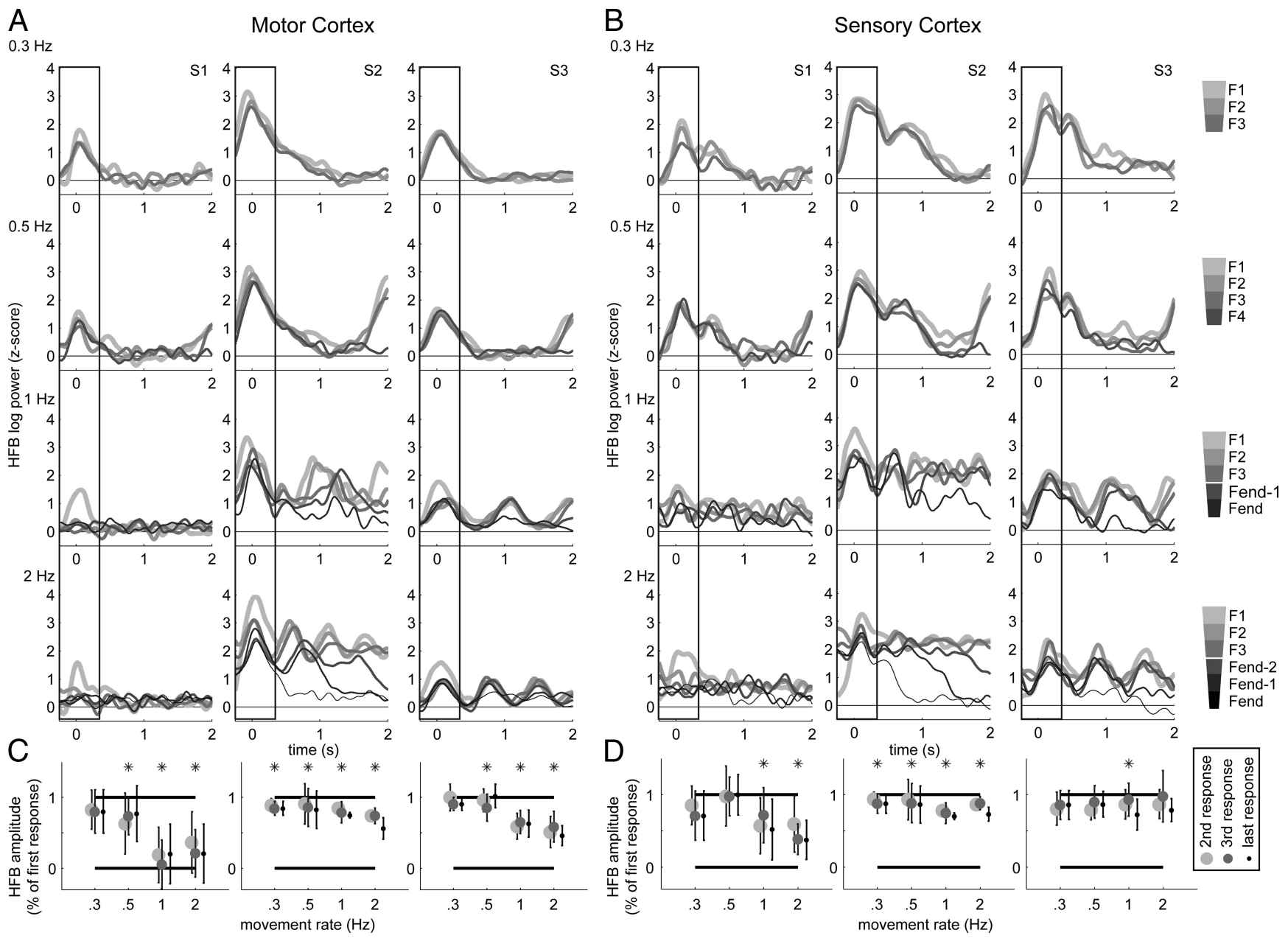

Figure 4. HFB responses on motor cortex and sensory cortex. A, HFB responses on motor cortex as a function of time after movement onset for subjects 1-3 (S1-S3) from left to right and during the different movement rates from top to bottom. The grayscale indicates the hand flexion number (F) as in Figure 2. The box accentuates the response related to onset of the current movement (at the faster rates with intercue interval of $<2$ s, the responses to the subsequent stimuli are also visible, but these are irrelevant for analysis). $\boldsymbol{B}$, HFB responses on sensory cortex for subjects $1-3$ from left to right and during the different movement rates from top to bottom. $C$, The relative change from the first response amplitude -100 to $300 \mathrm{~ms}$ around movement onset during the second, third, and last responses in motor cortex during the four different repetition tasks $(0.3,0.5,1$, and $2 \mathrm{~Hz}$ ). An * above the task denoted that flexion number had a significant effect on HFB amplitude ( $p<$ 0.05 by a repeated-measures ANOVA). $\boldsymbol{D}$, The same results but in sensory cortex. Note that, during the faster repetition tasks, the second, third, and last responses are suppressed. Note also that this suppression is more pronounced on motor cortex compared with sensory cortex.

based on Fig. $1 B$ ). Figure 4, $C$ and $D$, shows the change in HFB amplitude with respect to the size of the first response. Similarly as in Figure 3, $A$ and $B$, a decrease in response size for subsequent movements can be noted, especially for the faster movement conditions of 1 and $2 \mathrm{~Hz}$. As shown in Figure 4C, a repeatedmeasures ANOVA indicated that, in motor cortex, this decrease in HFB amplitude with subsequent movements was significant in one subject for the $0.3 \mathrm{~Hz}$ task and for all three subjects in the 0.5 , 1 , and $2 \mathrm{~Hz}$ tasks. In sensory cortex (Fig. 4D), a repeatedmeasures ANOVA indicated that the decrease in HFB amplitude with subsequent movements was significant in one subject for the 0.3 and $0.5 \mathrm{~Hz}$ tasks, for all three subjects in the $1 \mathrm{~Hz}$ task, and for two subjects in the $2 \mathrm{~Hz}$ task. With faster movement repetition rate, there is thus a clear decrease in HFB amplitude after the first response; in sensory cortex, this effect is similar but less pronounced.

To test whether HFB response amplitude might depend on the degree of beta suppression, we correlated the beta power -700 to -300 ms before movement onset with the HFB amplitude across trials. With the fast movement rates, there was a significant positive correlation between prestimulus beta and HFB amplitude across all subjects in motor cortex, but this was not the case in sensory cortex. It is not surprising that there was only a significant positive correlation in the fast movement conditions, because premovement beta does not enter the suppressed regimen in the slow conditions. In general, in trials in which beta is suppressed before movement onset, there is less HFB activity related to the movement.

\section{Is the change in the high-frequency response with repeated movement linear or nonlinear?}

Next, we assessed the nature of the repetition effect by fitting a linear and a nonlinear model to the data. Figure 5 shows the HFB amplitude as a function of time. Two different models were fitted to the HFB response sizes as a function of time. The first linear model was of the form $Y_{\mathrm{HFB}}=b t+c$, and the second nonlinear model was of the form $Y_{\mathrm{HFB}}=a(1 / t)+b t+c$, where $t$ is the time of movement onset after the first cue, and $Y$ is the HFB amplitude of each movement. Other nonlinear models could be fitted; this one was chosen because it converges to the linear function and has a sharp, nonlinear decrease in case $a$ is positive, but except for its nonlinearity, we do not attempt to draw any conclusions about 

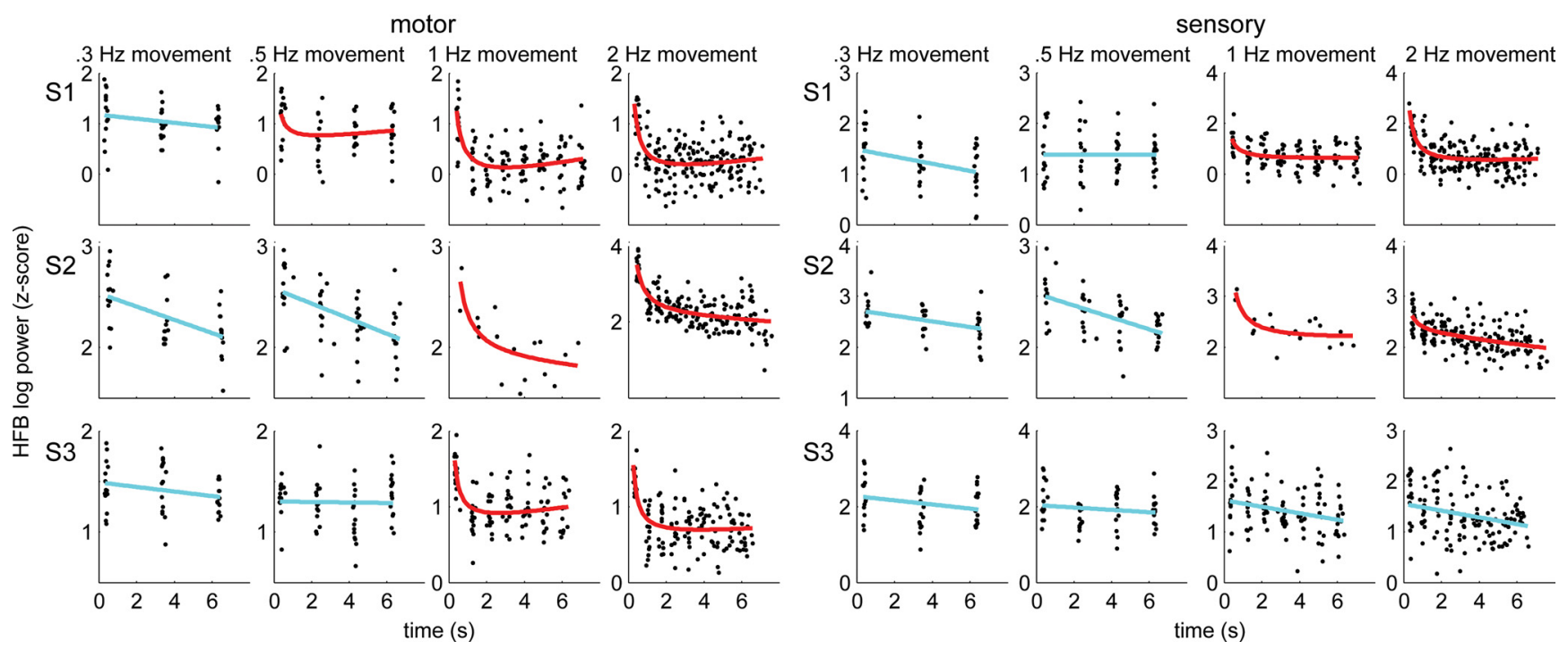

Figure 5. Fitting a linear or nonlinear model to the HFB change. The gray dots in every single plot display the HFB amplitude during one movement on the $y$-axis and the timing of that movement on the $x$-axis. Subjects (S1-S3) are shown in rows and the four different movement repetition tasks in columns; the average of the electrodes on motor cortex is shown on the left, the sensory cortex on the right. The linear model $\left(Y_{\text {HFB }}=b t+c\right)$ fitted to the data is shown in the cyan line, but if the nonlinear model $\left[Y_{\text {HFB }}=a(1 / t)+b t+c\right]$ fitted significantly better $(p<0.05$ by an $F$ test), a red line shows the nonlinear model.

the actual shape of the nonlinear decrease. To test whether the nonlinear model explained the data better, an $F$ test was performed on the difference in explained variance between the two models adjusted for the number of parameters $\left(\Delta R_{\text {adjusted }}^{2}\right)$. In all subjects, in the faster 1 and $2 \mathrm{~Hz}$ movement tasks, the motor cortex HFB amplitude was better explained by a nonlinear model compared with a linear model ( $p<0.01$ for all subjects; Fig. 5). In sensory cortex, the nonlinear model explained the data during the faster 1 and $2 \mathrm{~Hz}$ movement tasks better than the linear model in the first two subjects ( $p<0.005$ for these two subjects). Only in one subject in motor cortex was the $0.5 \mathrm{~Hz}$ movement better explained by a nonlinear model $(p<0.05)$. This indicates that, in motor cortex, there is a significant, nonlinear suppression of high-frequency activity with repeated movements at higher rates that converges to a linear state.

It could potentially be argued that the more accurate fit of the linear model for the slow movements with $\leq 0.5 \mathrm{~Hz}$ frequency was attributable to the small number of movements (three or four) rather than the decreased repetition rate, and there were simply not enough time points recorded for saturation to occur and nonlinearity to emerge. To verify this issue, we included only the first three or four movements from the more rapid rates. Even if only the first three or four movements were used, the nonlinear model still fit the data better than the nonlinear model for the fast movements. This emphasized that the main nonlinear effect in HFB amplitude for the rapid rates was the decrease after the first movement, and instead of using the model we chose, a step function could similarly be used.

\section{Spatial distribution of nonlinear decrease during fast movement}

The decrease in HFB response amplitude in the previous analysis was based on the averaged HFB response across electrodes on motor cortex and sensory cortex. We further explored whether this decrease was seen across a large number of individual electrodes on motor and sensory areas. The nonlinear regression model $\left[Y_{\mathrm{HFB}}=a(1 / t)+b t+c\right]$ was fitted for each electrode individually, and spatial distribution of the nonlinear regression coefficient, $a$, is shown in Figure 6. A positive regression coefficient, indicating that the HFB response shows a nonlinear decrease, can be noted for the faster tasks. Using the $F$ test approach as explained above, a significant increase (Bonferroni's corrected for the number of electrodes) in the model fit was found for 0,7 , 47 , and $70 \%$ of the electrodes in motor cortex after adding the nonlinear parameter for the $0.3,0.5,1$, and $2 \mathrm{~Hz}$ tasks on average across the three subjects, respectively. For sensory cortex, the nonlinear model improved the fit in $0,3,26$, and $33 \%$ of electrodes.

\section{Discussion}

In this study, neuronal population activity in sensorimotor cortex was assessed during hand movement (flexion-extension) at different rates to understand whether the link between neuronal activity and behavior is maintained with repetitive actions. We found that high-frequency (HFB) power exhibits a transient increase (response) in motor and somatosensory cortex during a movement and that this response, which reflects neuronal population activity, decreases after the initial movement at rates $>1$ $\mathrm{Hz}$. This decrease was nonlinear: after activity related to the initial movement, activity dropped significantly for the subsequent repeated movement and then converges. In motor cortex, the nonlinear decrease in response size with faster movement rates was noted for a large percentage of electrodes and not in a small subset. Since broadband high-frequency power changes have been linked to population firing rate (Manning et al., 2009; Miller et al., 2009a; Ray and Maunsell, 2011), this suggests that, after movement initiation, fewer motor cortex neurons have to fire or firing rates decline when multiple similar movements are made at a fast rate.

Sensory cortex showed some suppression with repetition that was not as strong as in motor cortex. In sensory cortex, this suppression can potentially be explained by accumulation of presynaptic inhibition of afferent input (Seki et al., 2003), which occurs with active movements. The suppression in motor cortex activity, which we observe during fast repetitive movements, cannot however be explained by this phenomenon. During the 
single-movement task, sensory responses differed from motor responses (Fig. 1). Although in motor cortex there was only one peak in the HFB response, sensory cortex showed two subsequent peaks. Cells on the postcentral gyrus are known to fire with two directions of movement (Fetz et al., 1980; Soso and Fetz, 1980), which may explain the two peaks in the sensory response. Surprisingly, our results showed that the first sensory response started before the onset of movement. Cells in sensory cortex can fire $\sim 60$ ms before movement onset, which is a similar time lag as shown in our results. Recent research has shown that sensory cortex may indeed be involved in motor control (Matyas et al., 2010), which may require some premovement activity. Whether the suppression we noted in sensory cortex can be fully explained by presynaptic inhibition or also partially by changes in the premovement motor control component has to be further investigated.

Although we found a significant decrease in high-frequency responses for subsequent movements at high repetition rates, we found no repetition effect for the beta-band response. However, beta-band suppression did not return to baseline at faster movement rates, in contrast with the slower movement rates. This agrees with a previous study with scalp EEG (Toma et al., 2002) and confirms that the phenomenon is present in primary sensorimotor cortex. Because previous studies reported decreased corticospinal excitability with high beta power (Hummel et al., 2002; Carroll et al., 2006), sustained suppression of beta-band activity may reflect a sustained release of inhibition. We indeed found a positive correlation between premovement beta and highfrequency amplitude. This correlation was apparent only during the fast movement tasks, which emphasizes that beta suppression may be a state in which inhibition is released rather than a continuum. It is possible that this release of inhibition may result in more efficient information processing, requiring less neuronal activity: ongoing beta oscillations only need to be suppressed during the first movement in the fast movement conditions, and initiation of the first movement may therefore require more activity than subsequent ones (Baker, 2007). This mechanism may explain why movements at slower rates are avoided (van der Wel et al., 2009) and fast movements are more efficiently processed.

Our results are important for the interpretation of fMRI and PET results during movement repetition. Previous fMRI and PET studies have found that increases in repetition rate are correlated with increased blood oxygen level-dependent (BOLD) activity and cerebral blood flow in motor areas, respectively, reaching a ceiling at fast repetition rates (Blinkenberg et al., 1996; Sadato et al., 1996, 1997; Turner et al., 1998; Wildgruber et al., 2001; Riecker et al., 2003). These studies generally assume that
Subject 2
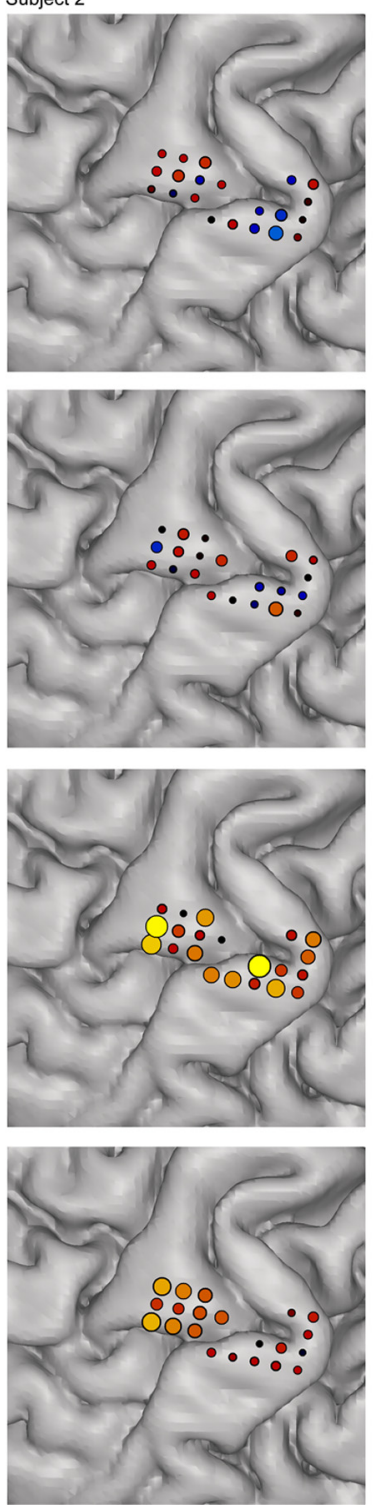

Subject 3
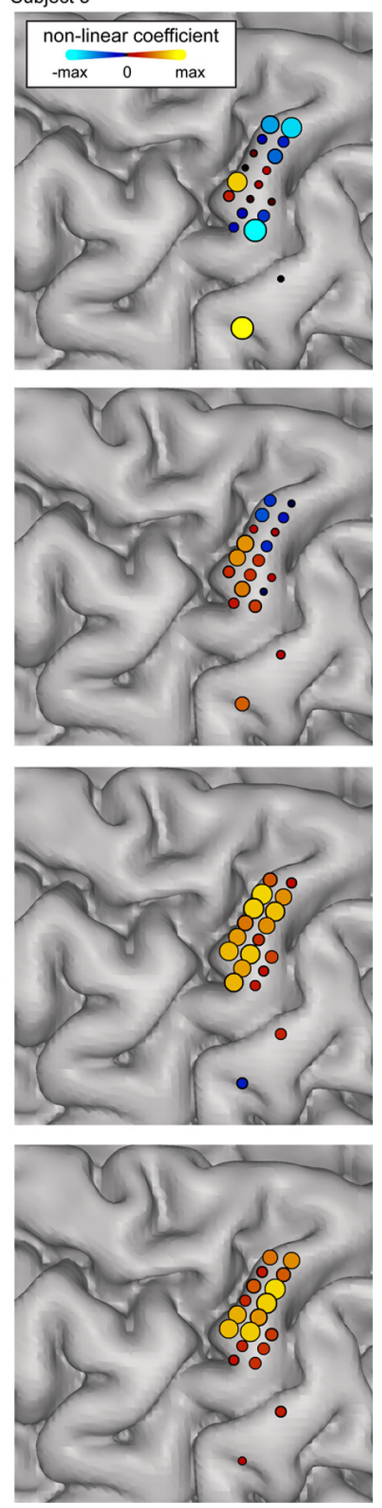

Figure 6. Spatial distribution of nonlinear coefficient. Spatial distribution of the amplitude of the nonlinear coefficient (a) from the model $Y_{\text {НFB }}=a(1 / t)+b t+c$. Subjects are shown in columns, and rows display different movement rates. Note that, at 1 and $2 \mathrm{~Hz}$, there is an overall increase in the nonlinear parameter, indicating that responses became nonlinearly reduced across the grid.

underlying neuronal activity is the same for repeated movements and often attribute the ceiling effects to nonlinearity in the BOLD response. Similarly, the duration of movement repetition affects the fMRI signal, which has also been attributed to nonlinear effects in the BOLD response (Birn et al., 2001). The BOLD response correlates well with high-frequency activity (Logothetis et al., 2001; Niessing et al., 2005; Lachaux et al., 2007; Hermes et al., 2011). Considering our results of nonlinear effects in highfrequency activity with faster movements, it may well be that the reported nonlinearity in the BOLD signal is overestimated. Hence, the relationship between neuronal activity and the vascular response ("neurovascular coupling") may be more linear than what has been reported.

It is important to understand the neurophysiology of repeated movements, because specific errors with movement initiation and perseveration are symptoms of several neurological disorders and in Parkinson's disease in particular (Ebersbach et al., 1994; Ridley, 1994; Praamstra et al., 1998; Redgrave et al., 2010). We 
showed that, for fast repeated movements, less primary motor cortex activity is generated after the initiation of the first movement. Also, after the first movement, there was a continuous power decrease of the beta rhythm, potentially indicating that the brain is in a different state. Interestingly, in subjects with Parkinson's disease, the suppression of the beta rhythm during movement is attenuated (Lim et al., 2006), and when deep brain stimulation alleviates the Parkinson's symptoms and normalizes the beta suppression, it is easier for these subjects to stop unwanted actions (Swann et al., 2011). Also, the onset of the beta decrease is delayed in these subjects (Magnani et al., 2002). Medication reduces this delay and also facilitates movement initiation. Parkinson's disease mainly affects subcortical structures, and beta rhythms have been related to subcortical-cortical interactions (Pfurtscheller and Lopes da Silva, 1999; Brown, 2003; Feige et al., 2005; Schnitzler et al., 2006; Jones et al., 2009). The combination of sustained beta desynchronization and a reduced high-frequency response after the initiation of repeated movements suggests a dynamic interplay between subcortical structures and motor cortex.

The suppression of high-frequency power effect could be associated with differences in hand movement. However, hand movement amplitude or speed did not show any significant differences with repetition. We were unable to obtain electromyographic measures, but because we did not notice significant differences in movement speed and subjects were not required to press against anything, it seems unlikely that force can explain this suppression effect. In addition, previous studies have not noticed any differences in electromyography between subsequent movements (Muthukumaraswamy, 2010) or movements made at different rates (Toma et al., 2002).

In conclusion, our data show that the relationship between motor actions and underlying neuronal activity is not consistent. The findings of repetition rate-dependent dynamics of neurophysiological parameters suggest that the amplitude of the HFB response in motor cortex may depend on the degree of synchronization of the beta rhythm. At slow rates, beta has time to return to baseline and a subsequent movement required a strong HFB response. At rates $\geq 1 \mathrm{~Hz}$, beta power remains depressed, and subsequent movements require a smaller HFB response.

\section{References}

Baker SN (2007) Oscillatory interactions between sensorimotor cortex and the periphery. Curr Opin Neurobiol 17:649-655.

Birn RM, Saad ZS, Bandettini PA (2001) Spatial heterogeneity of the nonlinear dynamics in the FMRI BOLD response. Neuroimage 14:817-826.

Blinkenberg M, Bonde C, Holm S, Svarer C, Andersen J, Paulson OB, Law I (1996) Rate dependence of regional cerebral activation during performance of a repetitive motor task: a PET study. J Cereb Blood Flow Metab 16:794-803.

Brown P (2003) Oscillatory nature of human basal ganglia activity: relationship to the pathophysiology of Parkinson's disease. Mov Disord 18:357-363.

Carroll TJ, Baldwin ER, Collins DF, Zehr EP (2006) Corticospinal excitability is lower during rhythmic arm movement than during tonic contraction. J Neurophysiol 95:914-921.

Crone NE, Miglioretti DL, Gordon B, Lesser RP (1998) Functional mapping of human sensorimotor cortex with electrocorticographic spectral analysis. II. Event-related synchronization in the gamma band. Brain 121:2301-2315.

Ebersbach G, Hättig H, Schelosky L, Wissel J, Poewe W (1994) Perseverative motor behaviour in Parkinson's disease. Neuropsychologia 32:799-804.

Feige B, Scheffler K, Esposito F, Di Salle F, Hennig J, Seifritz E (2005) Cortical and subcortical correlates of electroencephalographic alpha rhythm modulation. J Neurophysiol 93:2864-2872.

Fetz EE, Finocchio DV, Baker MA, Soso MJ (1980) Sensory and motor re- sponses of precentral cortex cells during comparable passive and active joint movements. J Neurophysiol 43:1070-1089.

Hermes D, Miller KJ, Noordmans HJ, Vansteensel MJ, Ramsey NF (2010) Automated electrocorticographic electrode localization on individually rendered brain surfaces. J Neurosci Methods 185:293-298.

Hermes D, Miller KJ, Vansteensel MJ, Aarnoutse EJ, Leijten FS, Ramsey NF (2011) Neurophysiologic correlates of fMRI in human motor cortex. Hum Brain Mapp. Advance online publication. Retrieved June 6, 2012. doi:10.1002/hbm. 21314

Hummel F, Andres F, Altenmüller E, Dichgans J, Gerloff C (2002) Inhibitory control of acquired motor programmes in the human brain. Brain 125:404-420

Jasper HH, Andrews HL (1938) Electro-encephalography III. Normal differentiation of occipital and precentral regions in man. Arch Neurol Psychiatry 39:96-115.

Jensen O, Goel P, Kopell N, Pohja M, Hari R, Ermentrout B (2005) On the human sensorimotor-cortex beta rhythm: sources and modeling. Neuroimage 26:347-355.

Jones SR, Pritchett DL, Sikora MA, Stufflebeam SM, Hämäläinen M, Moore CI (2009) Quantitative analysis and biophysically realistic neural modeling of the MEG mu rhythm: rhythmogenesis and modulation of sensory-evoked responses. J Neurophysiol 102:3554-3572.

Kubánek J, Miller KJ, Ojemann JG, Wolpaw JR, Schalk G (2009) Decoding flexion of individual fingers using electrocorticographic signals in humans. J Neural Eng 6:066001

Lachaux JP, Fonlupt P, Kahane P, Minotti L, Hoffmann D, Bertrand O, Baciu M (2007) Relationship between task-related gamma oscillations and BOLD signal: new insights from combined fMRI and intracranial EEG. Hum Brain Mapp 28:1368-1375.

Levy-Tzedek S, Tov MB, Karniel A (2011) Early switching between movement types: indication of predictive control? Brain Res Bull 85:283-288.

Lim VK, Hamm JP, Byblow WD, Kirk IJ (2006) Decreased desychronisation during self-paced movements in frequency bands involving sensorimotor integration and motor functioning in Parkinson's disease. Brain Res Bull 71:245-251

Logothetis NK, Pauls J, Augath M, Trinath T, Oeltermann A (2001) Neurophysiological investigation of the basis of the fMRI signal. Nature 412:150-157.

Magnani G, Cursi M, Leocani L, Volont é MA, Comi G (2002) Acute effects of L-dopa on event-related desynchronization in Parkinson's disease. Neurol Sci 23:91-97.

Manning JR, Jacobs J, Fried I, Kahana MJ (2009) Broadband shifts in LFP power spectra are correlated with single-neuron spiking in humans. J Neurosci 29:13613-13620.

Matyas F, Sreenivasan V, Marbach F, Wacongne C, Barsy B, Mateo C, Aronoff R, Petersen CC (2010) Motor control by sensory cortex. Science 330:1240-1243.

Miall RC, Ivry R (2004) Moving to a different beat. Nat Neurosci 7:1025-1026.

Miller KJ, Leuthardt EC, Schalk G, Rao RP, Anderson NR, Moran DW, Miller JW, Ojemann JG (2007) Spectral changes in cortical surface potentials during motor movement. J Neurosci 27:2424-2432.

Miller KJ, Sorensen LB, Ojemann JG, den Nijs M (2009a) Power-Law Scaling in the Brain Surface Electric Potential. PLoS Comput Biol 5:e1000609.

Miller KJ, Zanos S, Fetz EE, den Nijs M, Ojemann JG (2009b) Decoupling the cortical power spectrum reveals real-time representation of individual finger movements in humans. J Neurosci 29:3132-3137.

Muthukumaraswamy SD (2010) Functional properties of human primary motor cortex gamma oscillations. J Neurophysiol 104:2873-2885.

Niessing J, Ebisch B, Schmidt KE, Niessing M, Singer W, Galuske RA (2005) Hemodynamic signals correlate tightly with synchronized gamma oscillations. Science 309:948-951.

Pfurtscheller G (1981) Central beta rhythm during sensorimotor activities in man. Electroencephalogr Clin Neurophysiol 51:253-264.

Pfurtscheller G, Lopes da Silva FH (1999) Event-related EEG/MEG synchronization and desynchronization: basic principles. Clin Neurophysiol 110:1842-1857.

Praamstra P, Stegeman DF, Cools AR, Horstink MW (1998) Reliance on external cues for movement initiation in Parkinson's disease. Evidence from movement-related potentials. Brain 121:167-177.

Ray S, Maunsell JH (2011) Different origins of gamma rhythm and highgamma activity in macaque visual cortex. PLoS Biol 9:e1000610. 
Redgrave P, Rodriguez M, Smith Y, Rodriguez-Oroz MC, Lehericy S, Bergman H, Agid Y, DeLong MR, Obeso JA (2010) Goal-directed and habitual control in the basal ganglia: implications for Parkinson's disease. Nat Rev Neurosci 11:760-772.

Ridley RM (1994) The psychology of perserverative and stereotyped behaviour. Prog Neurobiol 44:221-231.

Riecker A, Wildgruber D, Mathiak K, Grodd W, Ackermann H (2003) Parametric analysis of rate-dependent hemodynamic response functions of cortical and subcortical brain structures during auditorily cued finger tapping: a fMRI study. Neuroimage 18:731-739.

Sadato N, Ibañez V, Deiber MP, Campbell G, Leonardo M, Hallett M (1996) Frequency-dependent changes of regional cerebral blood flow during finger movements. J Cereb Blood Flow Metab 16:23-33.

Sadato N, Ibañez V, Campbell G, Deiber MP, Le Bihan D, Hallett M (1997) Frequency-dependent changes of regional cerebral blood flow during finger movements: functional MRI compared to PET. J Cereb Blood Flow Metab 17:670-679.

Schaal S, Sternad D, Osu R, Kawato M (2004) Rhythmic arm movement is not discrete. Nat Neurosci 7:1136-1143.

Schnitzler A, Timmermann L, Gross J (2006) Physiological and pathological oscillatory networks in the human motor system. J Physiol Paris 99:3-7.

Seki K, Perlmutter SI, Fetz EE (2003) Sensory input to primate spinal cord is presynaptically inhibited during voluntary movement. Nat Neurosci 6:1309-1316.

Soso MJ, Fetz EE (1980) Responses of identified cells in postcentral cortex of awake monkeys during comparable active and passive joint movements. J Neurophysiol 43:1090-1110.

Swann N, Tandon N, Canolty R, Ellmore TM, McEvoy LK, Dreyer S, DiSano M, Aron AR (2009) Intracranial EEG reveals a time- and frequencyspecific role for the right inferior frontal gyrus and primary motor cortex in stopping initiated responses. J Neurosci 29:12675-12685.

Swann N, Poizner H, Houser M, Gould S, Greenhouse I, Cai W, Strunk J, George J, Aron AR (2011) Deep brain stimulation of the subthalamic nucleus alters the cortical profile of response inhibition in the beta frequency band: a scalp EEG study in Parkinson's disease. J Neurosci 31:5721-5729.

Toma K, Mima T, Matsuoka T, Gerloff C, Ohnishi T, Koshy B, Andres F, Hallett M (2002) Movement rate effect on activation and functional coupling of motor cortical areas. J Neurophysiol 88:3377-3385.

Turner RS, Grafton ST, Votaw JR, Delong MR, Hoffman JM (1998) Motor subcircuits mediating the control of movement velocity: a PET study. J Neurophysiol 80:2162-2176.

van Beijsterveldt CE, Molenaar PC, de Geus EJ, Boomsma DI (1996) Heritability of human brain functioning as assessed by electroencephalography. Am J Hum Genet 58:562-573.

van der Wel RP, Sternad D, Rosenbaum DA (2009) Moving the arm at different rates: slow movements are avoided. J Mot Behav 42:29-36.

Wildgruber D, Ackermann H, Grodd W (2001) Differential contributions of motor cortex, basal ganglia, and cerebellum to speech motor control: effects of syllable repetition rate evaluated by fMRI. Neuroimage 13:101109. 